\title{
Management of Ophthalmic Complications Secondary to Functional Endoscopic Sinus Surgery
}

Betül TUĞCU¹, Bilge ARAZ-ERSAN², Ercan INNCi³, Gökhan GÜLKILIK ${ }^{4}$

${ }^{1}$ Department of Ophthalmology, Bezmialem Vakif University Faculty of Medicine, Istanbul, Turkey

${ }^{2}$ Clinic of Ophthalmology, Kocaeli Derince Training and Research Hospital, Kocaeli, Turkey

${ }^{3}$ Clinic of Radiology, Bakırköy Dr. Sadi Konuk Education and Research Hospital, Istanbul, Turkey

${ }^{4}$ Department of Ophthalmology, Medipol University Faculty of Medicine, Istanbul, Turkey

\section{ABSTRACT}

Orbital complications of functional endoscopic sinus surgery (FESS) may cause irreversible damage to the orbital structures, including optic nerve, extraocular muscles, and lacrimal drainage system. The clinical diagnosis and management of these patients may be extremely difficult because of the complexity and severity of the injuries. We describe a patient with ocular motility disturbances, anisocoria, and optic neuropathy in the right eye secondary to FESS. Early medical and surgical intervention resulted in symptom resolution.

Keywords: Orbital complication, endoscopic sinus surgery, botulinum toxin

\section{Introduction}

A 36-year-old woman who underwent functional endoscopic sinus surgery (FESS) for chronic sinusitis had diplopia, anisocoria, and blurred vision in the right eye (OD) in immediate postoperative period. Visual acuity (VA) was 20/32 in the OD and pupillary direct light reflex was absent. The visual field (VF) testing revealed superior altitudinal scotoma in the OD. Ocular motility was almost completely limited in adduction, and it was partially limited in supra- and infraduction. The condition was consistent with injury to the optic nerve and inferior branch of the third cranial nerve.

\section{Case Presentation}

The patient underwent urgent revision of FESS with optic nerve decompression. Optic nerve compression by soft tissues and bone fragments were identified by the fracture of the lamina papyracea at the postethmoid level and partial transection of the medial rectus (MR) muscle. Oral corticosteroid treatment was initiated (1 mg/kg prednisolone). Postoperatively, an exotropia of 35 prism diopters in the OD (Fig 1a) was observed with an extreme limitation of adduction that precluded the eye from moving toward midline (Fig 1b). The VA improved to 20/20, but there was a VF defect at the right superonasal quadrant. The right pupil did not constrict to direct light or a near target, and the left pupil constricted normally. In dim light, the pupillary diameters were $7 \mathrm{~mm}$ in the OD and $3 \mathrm{~mm}$ in the OS. The application of $0.1 \%$ pilocarpine caused constriction of the pupil, thereby revealing tonic pupil. In control MRI, there was a contrast-enhanced inflammatory lesion at the posteromedial part of the orbital (Fig 2a). The dynamic MRI showed an absence of MR muscle function and injury to the inferior retroequatorial part of the muscle (Fig 2b). The forced duction test was positive with minimal uniform restriction in adduction, and the forced generation test revealed minimal muscle contractility. These findings suggested combined restriction and palsy of the MR muscle. One month after FESS, there was no improvement in ocular motility, and $5 \mathrm{U}$ of botulinum toxin type-A (Botox ${ }^{\circ}$; Allergan Inc., Irvine, CA) was injected into the right lateral rectus (LR) muscle under EMG guidance. Diplopia resolved 2 weeks later, and the patient was orthophoric in the OD. At the 3-month follow-up, the inflammatory lesion had 
resolved (Fig 2c). The patient was isocoric, orthophoric, and diplopia-free with minimal limitation of adduction of the OD (Figs 3a, 3b). Written informed consent was obtained from the patient for the publication of this case report and any accompanying images.

\section{Discussion}

The most catastrophic ophthalmic complication of FESS is injury to the optic nerves (1-3). The optic nerve may be injured directly or indirectly. In our case, the injury was indirect because of compression; after emergent orbital decompression, optic neuropathy resolved.

Anisocoria is infrequently encountered after endoscopic sinus surgery $(2,4,5)$. Surgical trauma to parasympathetic fibers of the third cranial nerve or the ciliary ganglion results in postoperative mydriasis. In the literature, resolution of mydriasis in 1-2 months after surgery was reported, and this condition was attributed to the postoperative perineural edema of the

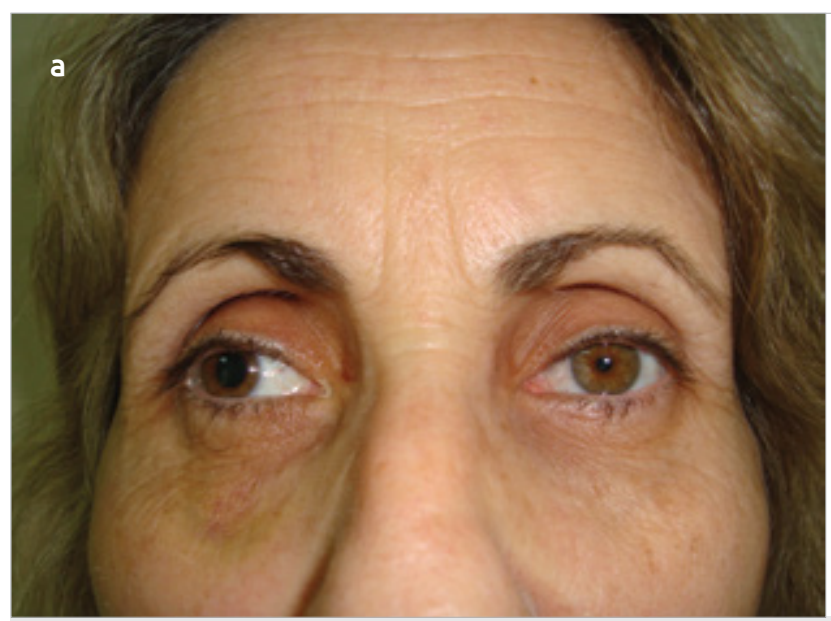

pupillomotor nerves $(4,5)$. Similar to these previous reports, in our patient, mydriasis resolved at the 3-month follow up.

Ocular motility disturbance is another rare complication of FESS (6). It can result from direct injury to the muscle or its nerve or to both. Clinical diagnosis may be extremely challenging. In particular, dynamic MRI is helpful in evaluating the function of the muscles by determining the changes in the sizes of the muscles in different gaze positions (7). In our case, dynamic MRI revealed paresis of the MR muscle and injury to the inferior retroequatorial part of the muscle.

Treatment for the motility complications of FESS is based on the type and severity of the injury. Management involving a partially transected muscle is still under debate. Huang et al. (8) reported that observation alone did not result in improvement, and that late repair led to little or no recovery. On the other hand, Hong et al. (9) reported that the treatment with Botox $^{\oplus}$ in the early period yielded improved outcomes over observation or surgery alone by preventing contraction of the antagonist muscle and more rapidly facilitating binocular

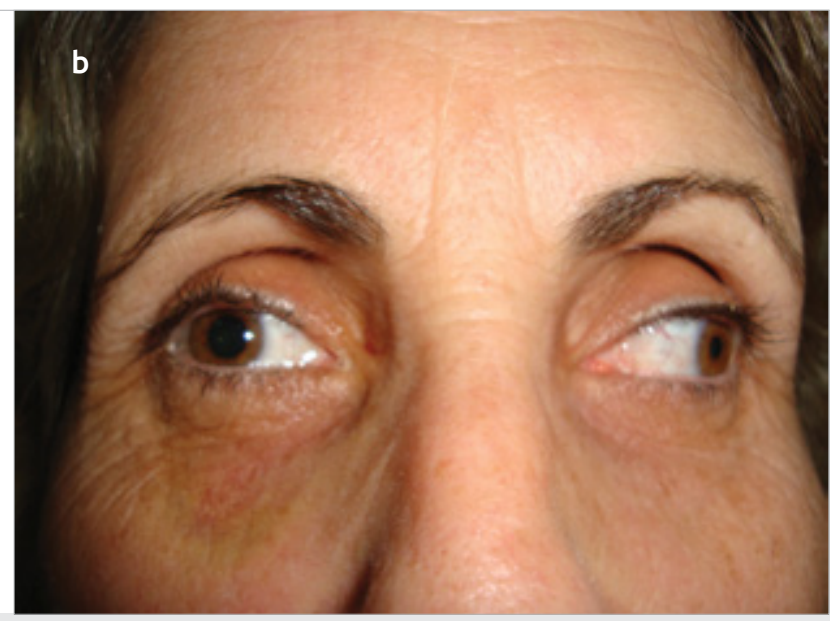

Figure 1. a,b. Initial clinical examination showing right exotropia and anisocoria (a). Marked limitation in adduction of the right eye (OD) (b).
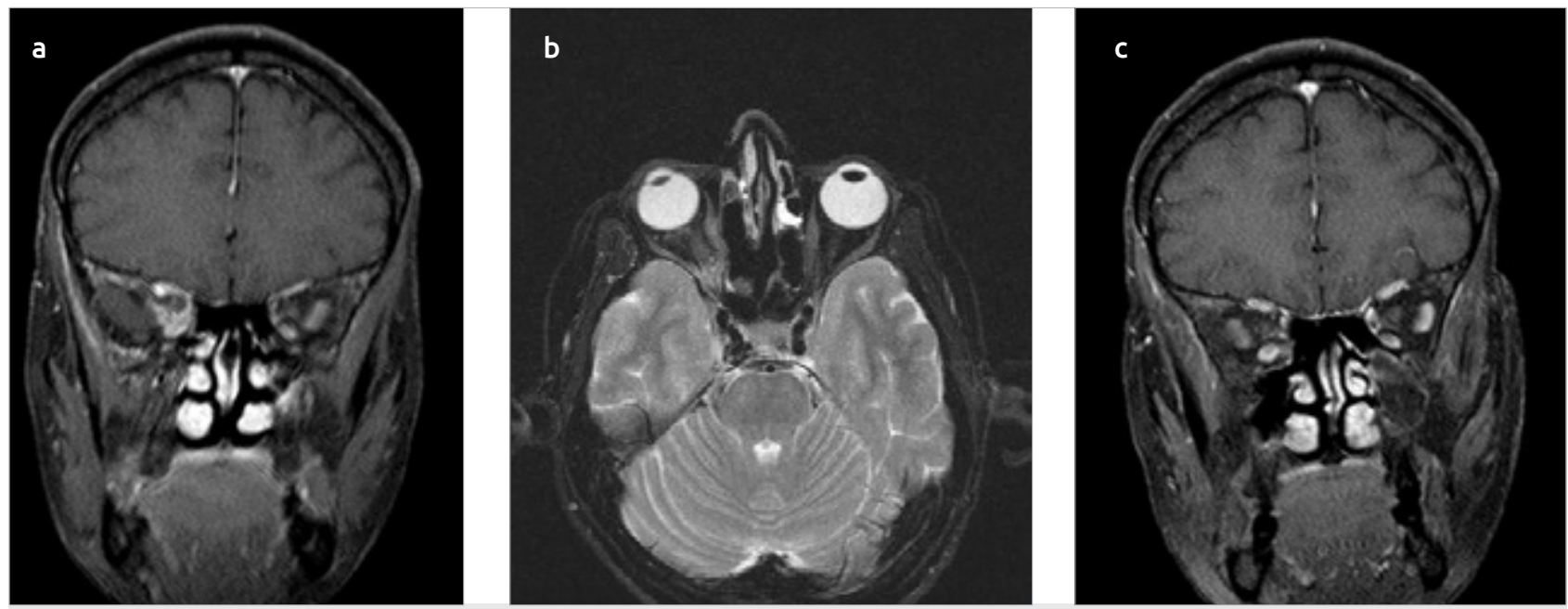

Figure 2. a-c. T1-weigted contrast-enhanced coronal image showing the inflammatory lesion at the posteromedial part of orbital (a). T2-weighted axial image demonstrating the tortuosity of the medial rectus (MR) muscle and loss of function (b). T1-weigted contrast-enhanced coronal image displaying the resolution of the lesion at the 3-month follow-up (c). 

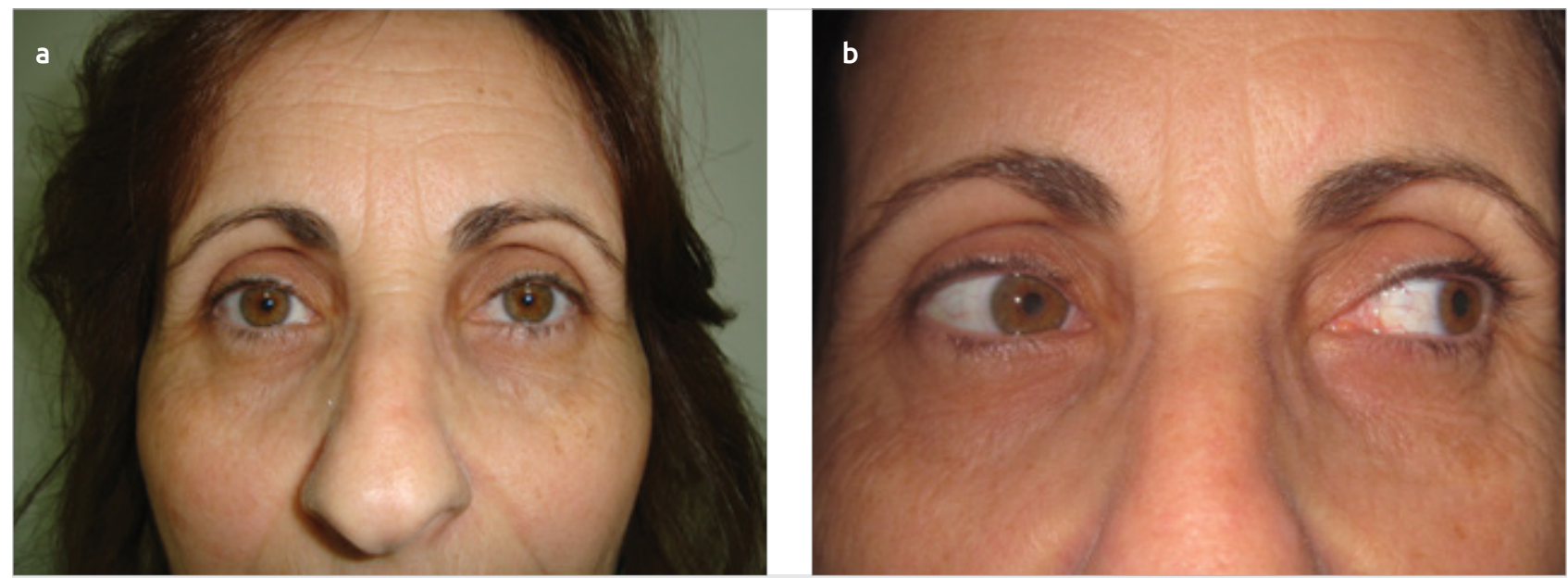

Figure 3. a,b. Clinical examination showing resolution of exotropia after BTA injection (a). Minimal limitation of adduction at 3-month follow-up (b).

single vision. In our case, after the injection of Botox ${ }^{\oplus}$ into the right LR muscle, diplopia was resolved and the patient remained orthophoric.

\section{Conclusion}

In conclusion, meticulous assessment is essential for the early recognition and management of orbital injuries and for improving the prognosis of these patients.

Informed Consent: Written informed consent was obtained from the patient/patients.

Peer-review: Externally peer-reviewed.

Author Contributions: Concept - B.T.; Design - B.T.; Supervision - B.T.; Data Collection and/or Processing - B.T., G.G., E.I..; Analysis and/or Interpretation - B.T., B.A., E.İ.; Literature Review - B.T., B.A., G.G., E.İ.; Writer - B.T., B.A., G.G., E.I.; Critical Review - B.T., B.A., E.İ.

Conflict of Interest: The authors declared no conflict of interest.

Financial Disclosure: The authors declared that this study has received no financial support.

\section{References}

1. Bhatti MT, Stankiewicz JA. Ophthalmic complications of endoscopic sinus surgery. Surv Ophthalmol 2003; 48: 389-402. [CrossRef]
2. Rene C, Rose GE, Lenthall R, Moseley I. Major orbital complications of endoscopic sinus surgery. Br J Ophthalmol 2001; 85: 598603. [CrossRef]

3. Wu W, Sia DI, Cannon PS, Selva D, Tu Y, Qu J. Visual acuity recovery in traumatic optic neuropathy following endoscopic optic nerve decompression: a case report. Ophthal Plast Reconstr Surg 2011; 27: e13-5. [CrossRef]

4. Kosko JR, Pratt MF, Chames M, Letterman I. Anisocoria: a rare consequence of endoscopic sinus surgery. Otolaryngol Head Neck Surg 1998; 118: 242-4. [CrossRef]

5. Bayramlar H, Miman MC, Demirel S. Inferior oblique paresis, mydriasis, and accommodative palsy as temporary complications of sinus surgery. J Neuroophthalmol 2004; 24: 225-7. [CrossRef]

6. Thacker NM, Velez FG, Demer JL, Rosenbaum AL. Strabismic complications following endoscopic sinus surgery: diagnosis and surgical management. J AAPOS 2004; 8: 488-94. [CrossRef]

7. Hall LS, McCann JD, Goldberg RA, et al. Strabismus after orbital fractures and sinus surgery. In: Rosenbaum AL, Santiago AP, eds. Clinical Strabismus Management. Principles and Surgical Techniques. Philadelphia, PA: W.B Saunders Co; 1999: 319-21.

8. Huang CM, Meyer DR, Patrinely JR, Soparkar CN, Dailey RA, Maus $\mathrm{M}$, et al. Medial rectus muscle injuries associated with functional endoscopic sinus surgery: characterization and management. Ophthal Plast Reconstr Surg 2003; 19: 25-37. [CrossRef]

9. Hong JE, Goldberg AN, Cockerham KP. Botulinum toxin A therapy for medial rectus injury during endoscopic sinus surgery. Am J Rhinol 2008; 22: 95-7.[CrossRef] 